\title{
On Some Transverse Geometrical Structures of Lifted Foliation to Its Conormal Bundle
}

\author{
Cristian Ida and Alexandru Oană \\ Department of Mathematics and Computer Science, Transilvania University of Braşov, Street Iuliu Maniu 50, 500091 Braşov, Romania
} Correspondence should be addressed to Cristian Ida; cristian.ida@unitbv.ro

Received 15 October 2014; Revised 9 February 2015; Accepted 20 February 2015

Academic Editor: Peter G. L. Leach

Copyright (C) 2015 C. Ida and A. Oană. This is an open access article distributed under the Creative Commons Attribution License, which permits unrestricted use, distribution, and reproduction in any medium, provided the original work is properly cited.

We consider the lift of a foliation to its conormal bundle and some transverse geometrical structures associated with this foliation are studied. We introduce a good vertical connection on the conormal bundle and, moreover, if the conormal bundle is endowed with a transversal Cartan metric, we obtain that the lifted foliation to its conormal bundle is a Riemannian one. Also, some transversally framed $f(3, \varepsilon)$-structures of corank 2 on the normal bundle of lifted foliation to its conormal bundle are introduced and an almost (para)contact structure on a transverse Liouville distribution is obtained.

\section{Introduction and Preliminaries}

The study of the lift of transversal Finsler foliations to their normal bundle using the technique of good vertical connection was initiated by Miernowski and Mozgawa [1] where it is proved that the lifted foliation is a Riemannian one. Also, using different methods, some connections between foliations and Lagrangians (or Hamiltonians) in order to recover Riemannian foliations are investigated in the recent papers [2-5]. Our aim in this paper is to extend the study from [1] for the case of lifted foliation to its conormal bundle. In this sense we introduce a good vertical connection on the conormal bundle and we give an application of it in order to obtain that the lifted foliation is a Riemannian one in the case when the conormal bundle is endowed with a transversal Cartan metric. Moreover, in this case, some transversally framed $f(3, \varepsilon)$-structures and an almost (para)contact structure associated with lifted foliation are investigated.

The methods used here are similarly and closely related to those used in $[1,6]$ for the case of transversal Finsler foliations.

Let us consider $M$ an $(n+m)$-dimensional manifold which will be assumed to be connected and orientable.

Definition 1. A codimension $n$ foliation $\mathscr{F}$ on $M$ is defined by a foliated cocycle $\left\{U_{i}, \varphi_{i}, f_{i, j}\right\}$ such that (i) $\left\{U_{i}\right\}, i \in I$, is an open covering of $M$;

(ii) for every $i \in I, \varphi_{i}: U_{i} \rightarrow N$ are submersions, where $N$ is an $n$-dimensional manifold, called transversal manifold;

(iii) the maps $f_{i, j}: \varphi_{i}\left(U_{i} \cap U_{j}\right) \rightarrow \varphi_{j}\left(U_{i} \cap U_{j}\right)$ satisfy

$$
\varphi_{j}=f_{i, j} \circ \varphi_{i}
$$

for every $(i, j) \in I \times I$ such that $U_{i} \cap U_{j} \neq \emptyset$.

Every fibre of $\varphi_{i}$ is called a plaque of the foliation. Condition (1) says that on the intersection $U_{i} \cap U_{j}$ the plaques defined, respectively, by $\varphi_{i}$ and $\varphi_{j}$ coincide. The manifold $M$ is decomposed into a family of disjoint immersed connected submanifolds of dimension $m$; each of these submanifolds is called a leaf of $\mathscr{F}$.

By $T \mathscr{F}$ we denote the tangent bundle to $\mathscr{F}$ and $\Gamma(\mathscr{F})$ is the space of its global sections, that is, vector fields tangent to $\mathscr{F}$, and by $Q \mathscr{F}=T M / T \mathscr{F}$ we denote the normal bundle of $\mathscr{F}$.

In this paper, a system of local coordinates adapted to the foliation $\mathscr{F}$ means coordinates $\left(x^{1}, \ldots, x^{n}, y^{1}, \ldots, y^{m}\right)$ on an open subset $U$ on which the foliation is trivial and defined by the equations $d x^{a}=0, a=1, \ldots, n$.

We notice that the total spaces of the conormal bundle $Q^{*} \mathscr{F}$ of $\mathscr{F}$ carry a natural foliation $\widetilde{\mathscr{F}}$ of codimension $2 n$ such 
that the leaves of $\widetilde{\mathscr{F}}$ are covering spaces of the leaves of $\mathscr{F}$, and it is called the natural lift of $\mathscr{F}$ to its conormal bundle $Q^{*} \mathscr{F}$.

If we denote by $\left\{d x^{a}\right\}, a=1, \ldots, n$, the corresponding local coframe on $Q^{*} \mathscr{F}$, then we can induce a chart $\left(x^{a}, p_{a}, y^{u}\right)$ on $Q^{*} \mathscr{F}$ where $p=p_{a} d x^{a} \in \Gamma\left(Q^{*} \mathscr{F}\right)$, and the system of equations $x^{a}=$ const., $p_{a}=$ const. defines the foliation $\widetilde{\mathscr{F}}$.

Let $Q \widetilde{\mathscr{F}}=T\left(Q^{*} \mathscr{F}\right) / T \widetilde{\mathscr{F}}$ be the normal bundle of the foliated manifold $\left(Q^{*} \mathscr{F}, \widetilde{\mathscr{F}}\right)$. The vectors $\left\{\partial / \partial x^{a}, \partial / \partial p_{a}\right\}$, $a=1, \ldots, n$, form a natural frame of $Q \widetilde{\mathscr{F}}$ at the point $\left(x^{a}, p_{a}, y^{u}\right) \in Q^{*} \mathscr{F}$. The canonical projection $\pi: Q^{*} \mathscr{F} \rightarrow$ $M$ given by $\pi\left(x^{a}, p_{a}, y^{u}\right)=\left(x^{a}, y^{u}\right)$ induces another projection $\pi_{*}: T\left(Q^{*} \mathscr{F}\right) \rightarrow T M$ which maps the vectors tangent to $\widetilde{\mathscr{F}}$ in the vectors tangent to $\mathscr{F}$. Thus, $\pi_{*}$ induces a mapping $\tilde{\pi}_{*}: Q \widetilde{\mathscr{F}} \rightarrow Q \mathscr{F}$ and is denoted by $V\left(Q^{*} \mathscr{F}\right)=\operatorname{ker} \tilde{\pi}_{*}$ which is a vertical bundle spanned by the vectors $\left\{\partial / \partial p_{a}\right\}$, $a=1, \ldots, n$.

Lemma 2. Let $o: M \rightarrow Q^{*} \mathscr{F}$ be the zero section of the conormal bundle $Q^{*} \mathscr{F}$. Then the set $o(M)$ is saturated on $Q^{*} \mathscr{F}$ with foliation $\widetilde{\mathscr{F}}$.

\section{Good Vertical Connection on $\left(Q^{*} \mathscr{F}, \widetilde{\mathscr{F}}\right)$}

The purpose of this section is to define a linear connection $\nabla: \mathscr{X}\left(V\left(Q^{*} \mathscr{F}\right)\right) \rightarrow \mathscr{X}\left(\mathrm{T}^{*}\left(\widetilde{\mathrm{Q}}^{*} \mathscr{F}\right) \otimes V\left(Q^{*} \mathscr{F}\right)\right)$ related to considered foliated structure, where $\widetilde{Q}^{*} \mathscr{F}=Q^{*} \mathscr{F}-o(M)$. Since we have the foliated manifold $\left(\widetilde{Q}^{*} \mathscr{F}, \widetilde{F}\right)$, we are looking for a Bott connection such that for any vector field $X$ tangent to $\widetilde{\mathscr{F}}$ and any transversal vector field $Y$ we have

$$
\nabla_{X} Y=p_{Q} \widetilde{\mathscr{F}}([X, \tilde{Y}]),
$$

where $p_{\mathrm{Q} \widetilde{F}}: T\left(Q^{*} \mathscr{F}\right) \rightarrow Q \widetilde{\mathscr{F}}$ is the canonical projection and $p_{\mathrm{Q} \widetilde{F}}(\widetilde{Y})=Y$.

Let us consider now the $\widetilde{\mathscr{F}}$-transversal Hamilton-Liouville vector field defined by $C^{*}: Q^{*} \mathscr{F} \rightarrow V\left(Q^{*} \mathscr{F}\right)$, $C^{*}\left(x^{a}, p_{a}, y^{u}\right)=p_{a}\left(\partial / \partial p_{a}\right)$. It can be checked that this definition is well posed. From the definition of the Bott connection, the following lemma holds.

Lemma 3. Let $\nabla: \mathscr{X}\left(V\left(Q^{*} \mathscr{F}\right)\right) \rightarrow \mathscr{X}\left(T^{*}\left(\widetilde{Q}^{*} \mathscr{F}\right) \otimes V\left(Q^{*} \mathscr{F}\right)\right)$ be a Bott connection. Then $\nabla_{X} C^{*}=0$ for every vector field tangent to $\widetilde{\mathscr{F}}$.

Now, consider the local frame $\left\{\partial / \partial x^{a}, \partial / \partial p_{a}, \partial / \partial y^{u}\right\}$ of $T\left(Q^{*} \mathscr{F}\right)$ and recall that the vectors $\left\{\partial / \partial p_{a}\right\}$ form the basis of $V\left(Q^{*} \mathscr{F}\right)$. With these settings we put

$$
\begin{gathered}
\nabla_{\partial / \partial x^{a}} \frac{\partial}{\partial p_{b}}=\Gamma_{a c}^{b} \frac{\partial}{\partial p_{c}}, \quad \nabla_{\partial / \partial p_{a}} \frac{\partial}{\partial p_{b}}=\Gamma_{c}^{a b} \frac{\partial}{\partial p_{c}}, \\
\nabla_{\partial / \partial y^{u}} \frac{\partial}{\partial p_{b}}=\Gamma_{u c}^{b} \frac{\partial}{\partial p_{c}} .
\end{gathered}
$$

From the above formulas it follows that

$$
\Gamma_{u c}^{b}=0, \quad \nabla_{\partial / \partial p_{a}} C^{*}=\left(\delta_{c}^{a}+p_{b} \Gamma_{c}^{a b}\right) \frac{\partial}{\partial p_{c}} .
$$

The Bott connection $\nabla$ allows us to define a mapping

$$
L: \mathscr{X}(Q \widetilde{\mathscr{F}}) \longrightarrow \mathscr{X}\left(V\left(Q^{*} \mathscr{F}\right)\right), \quad L(X)=\nabla_{\widetilde{X}} C^{*}
$$

where $p_{\mathrm{Q} \widetilde{F}}(\widetilde{X})=X$. If we denote by $\Lambda$ the restriction of the linear mapping $L$ to the bundle $V\left(Q^{*} \mathscr{F}\right)$, then we can state the following.

Definition 4. The Bott connection $\nabla$ is said to be a good vertical connection if $\Lambda: V\left(Q^{*} \mathscr{F}\right) \rightarrow V\left(Q^{*} \mathscr{F}\right)$ is a bundle isomorphism.

Observe that $\nabla$ is a good vertical connection if and only if the matrix $\delta_{c}^{a}+p_{b} \Gamma_{c}^{a b}$ is nondegenerated. If we put $H\left(Q^{*} \mathscr{F}\right)=$ $\operatorname{ker} L$, then we can split the bundle $Q \widetilde{\mathscr{F}}$ into direct sum:

$$
Q \widetilde{\mathscr{F}}=H\left(Q^{*} \mathscr{F}\right) \oplus V\left(Q^{*} \mathscr{F}\right) .
$$

The coefficients of the mapping $L$ in the basis $\left\{\partial / \partial x^{a}, \partial / \partial p_{a}\right\}$ of $Q \widetilde{\mathscr{F}}$ are

$$
\begin{gathered}
L\left(\frac{\partial}{\partial x^{a}}\right)=p_{b} \Gamma_{a c}^{b} \frac{\partial}{\partial p_{c}} \\
L\left(\frac{\partial}{\partial p_{a}}\right)=\left(\delta_{c}^{a}+p_{b} \Gamma_{c}^{a b}\right) \frac{\partial}{\partial p_{c}}=L_{c}^{a} \frac{\partial}{\partial p_{c}} .
\end{gathered}
$$

It is easy to check that the vectors $\delta / \delta x^{a}=\partial / \partial x^{a}+$ $N_{a b}\left(\partial / \partial p_{b}\right)$, where $N_{a b}=-\left(L^{-1}\right)_{b}^{c} p_{d} \Gamma_{a c}^{d}$, form a basis of $\operatorname{ker} L$. In the sequel we will use the basis $\left\{\delta / \delta x^{a}, \partial / \partial p_{a}\right\}$, called adapted, as well as its dual $\left\{d x^{a}, \delta p_{a}=d p_{a}-N_{a b} d x^{b}\right\}$. Using this coframe we can define the local connection forms by

$$
\nabla \frac{\partial}{\partial p_{b}}=\omega_{a}^{b} \otimes \frac{\partial}{\partial p_{a}}
$$

where

$$
\begin{aligned}
\omega_{a}^{b} & =\Gamma_{c a}^{b} d x^{c}+\Gamma_{a}^{b c} d p_{c}=\left(\Gamma_{c a}^{b}+\Gamma_{a}^{b d} N_{d c}\right) d x^{c}+\Gamma_{a}^{b c} \delta p_{c} \\
& =H_{c a}^{b} d x^{c}+\Gamma_{a}^{b c} \delta p_{c} .
\end{aligned}
$$

Notice that $H_{c a}^{b} p_{b}=N_{c a}$. The formula $\theta\left(\partial / \partial p_{a}\right)=\delta / \delta x^{a}$ defines a linear mapping $\theta: V\left(Q^{*} \mathscr{F}\right) \rightarrow H\left(Q^{*} \mathscr{F}\right)$. This mapping allows us to extend the connection $\nabla$ to the horizontal bundle $H\left(Q^{*} \mathscr{F}\right)$ by

$$
\nabla_{X} Y=\theta\left(\nabla_{X} \theta^{-1}(Y)\right)
$$

where $Y \in \Gamma\left(H\left(Q^{*} \mathscr{F}\right)\right), X \in \Gamma\left(T\left(Q^{*} \mathscr{F}\right)\right)$. In this way we construct a linear connection in $Q \widetilde{\mathscr{F}}$ :

$$
\nabla_{X} Y=\nabla_{X}(v(Y))+\nabla_{X}(Y-v(Y)),
$$

where $Y \in \Gamma(Q \widetilde{\mathscr{F}}), X \in \Gamma\left(T\left(Q^{*} \mathscr{F}\right)\right)$ and $v: Q \widetilde{\mathscr{F}} \rightarrow$ $V\left(Q^{*} \mathscr{F}\right)$ is the vertical projection from decomposition (6). In particular we have

$$
\nabla \frac{\delta}{\delta x^{a}}=\omega_{a}^{b} \otimes \frac{\delta}{\delta x^{b}}
$$

where $\omega_{a}^{b}$ is given in (9). 
If $\varphi \in \Gamma\left(Q^{*} \widetilde{\mathscr{F}} \otimes Q \widetilde{\mathscr{F}}\right)$ is a 1 -form with values in $Q \widetilde{\mathscr{F}}$, locally given by

$$
\varphi=\varphi^{a} \otimes \frac{\delta}{\delta x^{a}}+\varphi_{b} \otimes \frac{\partial}{\partial p_{b}},
$$

then, following $[1,7]$, we can define an exterior differential $D \varphi$ by putting

$$
D \varphi=\left(d \varphi^{a}-\varphi^{a} \wedge \omega_{a}^{c}\right) \otimes \frac{\delta}{\delta x^{a}}+\left(d \varphi_{b}-\varphi_{b} \wedge \omega_{c}^{b}\right) \otimes \frac{\partial}{\partial p_{c}} .
$$

A straightforward calculus shows that the above formula is well defined. by

The bundle $Q^{*} \widetilde{\mathscr{F}} \otimes Q \widetilde{\mathscr{F}}$ admits a natural section $\eta$ given

$$
\eta=d x^{a} \otimes \frac{\partial}{\partial x^{a}}+d p_{b} \otimes \frac{\partial}{\partial p_{b}}=d x^{a} \otimes \frac{\delta}{\delta x^{a}}+\delta p_{b} \otimes \frac{\partial}{\partial p_{b}} .
$$

It is clear that the form $\eta$ is well defined.

Definition 5. The form $\zeta=D \eta$ is called the torsion form of the connection $\nabla$.

Locally the form $\zeta$ can be expressed as follows:

$$
\begin{aligned}
D \eta & =\left(-d x^{a} \wedge \omega_{a}^{c}\right) \otimes \frac{\delta}{\delta x^{c}}+\left(d\left(\delta p_{b}\right)-\delta p_{c} \wedge \omega_{b}^{c}\right) \otimes \frac{\partial}{\partial p_{b}} \\
& =\zeta^{c} \otimes \frac{\delta}{\delta x^{c}}+\zeta_{b} \otimes \frac{\partial}{\partial p_{b}},
\end{aligned}
$$

where

$$
\begin{gathered}
\zeta^{c}=\frac{1}{2}\left(H_{e a}^{c}-H_{a e}^{c}\right) d x^{a} \wedge d x^{e}-\Gamma_{a}^{c e} d x^{a} \wedge \delta p_{e} \\
\zeta_{b}=-d N_{c b} \wedge d x^{c}-H_{e b}^{a} \delta p_{a} \wedge d x^{e}-\Gamma_{b}^{a e} \delta p_{a} \wedge \delta p_{e} .
\end{gathered}
$$

\section{Transversal Cartan Metrics on $Q^{*} \mathscr{F}$ and Riemannian Foliations}

As in the case of transversal Finsler metrics on the normal bundle of a foliation, $[1,3]$, a transversal Cartan metric on $Q^{*} \mathscr{F}$ is a basic function (with respect to the lifted foliation $\widetilde{\mathscr{F}}) K: Q^{*} \mathscr{F} \rightarrow[0, \infty)$ which has the following properties:

(i) $K$ is $C^{\infty}$ on $\widetilde{Q}^{*} \mathscr{F}$;

(ii) $K(x, \lambda p)=\lambda K(x, p)$ for all $\lambda>0$;

(iii) the $n \times n$ matrix $\left(g^{a b}\right)$, where $g^{a b}=$ $(1 / 2)\left(\partial^{2} K^{2} / \partial p_{a} \partial p_{a}\right)$, is positive definite at all points of $\widetilde{Q}^{*} \mathscr{F}$.

Also $K(x, p)>0$, whenever $p \neq 0$. As usual, [8], the properties of $K$ imply that

$$
\begin{aligned}
p^{a}=g^{a b} p_{b}, \quad p_{a} & =g_{a b} p^{b}, \quad K^{2}=g^{a b} p_{a} p_{b}=p_{a} p^{a}, \\
C^{a b c} p_{c} & =C^{a c b} p_{c}=C^{c a b} p_{c}=0,
\end{aligned}
$$

where $\left(g_{a b}\right)$ is the inverse matrix of $\left(g^{b a}\right)$ and we have put $p^{a}=(1 / 2)\left(\partial K^{2} / \partial p_{a}\right), C^{a b c}=-(1 / 4)\left(\partial^{3} K^{2} / \partial p_{a} \partial p_{b} \partial p_{c}\right)$.

Also, $g^{a b}$ determines a metric structure on $V\left(Q^{*} \mathscr{F}\right)$ by setting

$$
G^{v}(X, Y)=g^{a b}(x, p) X_{a}(x, y, p) Y_{b}(x, y, p),
$$

for every $X=X_{a}(x, y, p)\left(\partial / \partial p_{a}\right)$ and $Y=Y_{b}(x, y, p)(\partial /$ $\left.\partial p_{b}\right) \in \Gamma\left(V\left(Q^{*} \mathscr{F}\right)\right)$.

Similar reasons as for transversal Finsler foliations (see Theorem 3.1 from [1]) lead to the following result.

Theorem 6. Let $K: Q^{*} \mathscr{F} \rightarrow[0, \infty)$ be a transversal Cartan metric and let $G^{v}$ be the Riemannian metric on $V\left(Q^{*} \mathscr{F}\right)$ induced by $K$ as in (19). Then there exists exactly one Bott vertical connection $\nabla: \mathscr{X}\left(V\left(Q^{*} \mathscr{F}\right)\right) \rightarrow \mathscr{X}\left(T^{*}\left(\widetilde{Q}^{*} \mathscr{F}\right) \otimes\right.$ $\left.V\left(Q^{*} \mathscr{F}\right)\right)$ such that

(i) $\nabla$ is a good vertical connection;

(ii) if $X, Y \in \Gamma\left(V\left(Q^{*} \mathscr{F}\right)\right)$ and $Z \in \Gamma\left(T\left(\widetilde{Q}^{*} \mathscr{F}\right)\right)$, then

$$
Z G^{v}(X, Y)=G^{v}\left(\nabla_{Z} X, Y\right)+G^{v}\left(X, \nabla_{Z} Y\right)
$$

(iii) $\zeta(X, Y)=0$ for every $X, Y \in \Gamma\left(V\left(Q^{*} \mathscr{F}\right)\right)$;

(iv) $\zeta(X, Y) \in \Gamma\left(V\left(Q^{*} \mathscr{F}\right)\right)$ for every $X, Y \in \Gamma\left(H\left(Q^{*} \mathscr{F}\right)\right)$.

Also, the isomorphism $\theta$ does not depend on the coordinates along the leaves of $\widetilde{F}$; so the Riemannian metric in $Q \widetilde{\mathscr{F}}$ defined by $G=G^{h}+G^{v}$, where $G^{h}(X, Y)=G^{v}\left(\theta^{-1}(X), \theta^{-1}(Y)\right)$ for every $X, Y \in \Gamma\left(H\left(Q^{*} \mathscr{F}\right)\right)$ and $G(X, Y)=0$ for every $X \in \Gamma\left(H\left(Q^{*} \mathscr{F}\right)\right)$ and $Y \in \Gamma\left(V\left(Q^{*} \mathscr{F}\right)\right)$, is a transversal Riemannian metric for the lifted foliation $\mathscr{F}$ to the conormal bundle $Q^{*} \mathscr{F}$ of $\mathscr{F}$. Hence, we can consider the following.

Theorem 7. If the conormal bundle of foliation $\mathscr{F}$ is endowed with a transversal Cartan metric, then the lifted foliation $\widetilde{F}$ to the conormal bundle $Q^{*} \mathscr{F}$ is Riemannian.

\section{Transversally Framed $f(3, \varepsilon)$-Structures on $\left(Q^{*} \mathscr{F}, \widetilde{\mathscr{F}}\right)$}

The study of structures on manifolds defined by a tensor field satisfying $f^{3} \pm f=0$ has the origin in a paper by Yano [9]. Later on, these structures have been generically called $f$-structures. On the tangent manifold of a Finsler space, the notion of framed $f(3,1)$-structure was defined and studied by Anastasiei in [10] and on the cotangent bundle of a Cartan space the study is continued in $[11,12]$. Taking into account that the conormal bundle $Q^{*} \mathscr{F}$ has a local model of a cotangent manifold, in this section we extend the study concerning $f$-structures in our context.

Let $\varepsilon= \pm 1$. A framed $f(3, \varepsilon)$-structure of corank $s$ on a $(2 n+s)$-dimensional manifold $N$ is a natural generalization of an almost contact structure on $N$ (for $\varepsilon=1$ ) and of an almost paracontact structure on $N$ (for $\varepsilon=-1$ ), respectively, and it is a triplet $\left(f,\left(\xi_{i}\right),\left(\omega^{i}\right)\right), i=1, \ldots, s$, where $f$ is a tensor 
field of type $(1,1),\left(\xi_{i}\right)$ are vector fields, and $\left(\omega^{i}\right)$ are 1-forms on $N$ such that

$$
\begin{gathered}
\omega^{i}\left(\xi_{j}\right)=\delta_{j}^{i}, \quad f\left(\xi_{i}\right)=0, \quad \omega^{i} \circ f=0, \\
f^{2}=-\varepsilon\left(I-\sum_{i} \omega^{i} \otimes \xi_{i}\right)
\end{gathered}
$$

where $I$ denotes the Kronecker tensor field on $N$. The name of $f(3, \varepsilon)$-structure was suggested by the identity $f^{3}+\varepsilon f=0$. For an account of such kind of structures, we refer to [13].

Let us consider now that $Q^{*} \mathscr{F}$ is endowed with a transversal Cartan metric $K$. The linear operator $\phi$ given in the local adapted basis of $Q \widetilde{\mathscr{F}}$ by

$$
\phi\left(\frac{\delta}{\delta x^{a}}\right)=-\varepsilon g_{a b} \frac{\partial}{\partial p_{b}}, \quad \phi\left(\frac{\partial}{\partial p_{a}}\right)=g^{a b} \frac{\delta}{\delta x^{b}}
$$

defines an almost complex structure on $Q \widetilde{F}$ for $\varepsilon=1$ and an almost paracomplex structure on $Q \widetilde{\mathscr{F}}$ for $\varepsilon=-1$, respectively. We also have

$$
G(\phi(X), \phi(Y))=G(X, Y), \quad \forall X, Y \in \Gamma(Q \widetilde{\mathscr{F}}) .
$$

Let us put $\xi_{1}=\left(p^{a} / K\right)\left(\delta / \delta x^{a}\right)$ and $\xi_{2}=\left(p_{a} / K\right)\left(\partial / \partial p_{a}\right)=$ $(1 / K) C^{*}$. Thus, we have two global transverse vector fields on $\left(Q^{*} \mathscr{F}, \widetilde{F}\right)$ which are linearly independent. The first is transversally horizontal and the second one is transversally vertical.

From the definition of $\phi$ it follows

$$
\phi\left(\xi_{1}\right)=-\varepsilon \xi_{2}, \quad \phi\left(\xi_{2}\right)=\xi_{1} .
$$

Now, if we consider the dual transverse 1 -forms of $\xi_{1}$ and $\xi_{2}$, respectively, locally given by $\omega^{1}=\left(p_{a} / K\right) d x^{a}$ and $\omega^{2}=$ $\left(p^{a} / K\right) \delta p_{a}$, then we easily check that

$$
\begin{gathered}
\omega^{1} \circ \phi=\omega^{2}, \quad \omega^{2} \circ \phi=-\varepsilon \omega^{1}, \\
\omega^{1}(X)=G\left(X, \xi_{1}\right), \quad \omega^{2}(X)=G\left(X, \xi_{2}\right), \quad \forall X \in \Gamma(Q \widetilde{F}) .
\end{gathered}
$$

Next, using $\phi, \xi_{i}$, and $\omega^{i}, i \in\{1,2\}$, we construct the transverse tensor field $f$ of type $(1,1)$ on $\left(Q^{*} \mathscr{F}, \widetilde{F}\right)$ by putting

$$
f(X)=\phi(X)-\omega^{2}(X) \xi_{1}+\varepsilon \omega^{1}(X) \xi_{2}, \quad X \in \Gamma(Q \widetilde{\mathscr{F}}) .
$$

Using a similar argument as in $[6,10-12]$ by direct calculus, we obtain the following.

Theorem 8. The triple $\left(f,\left(\xi_{i}\right),\left(\omega^{i}\right)\right), i \in\{1,2\}$, provides some transversally framed $f(3, \varepsilon)$-structures of corank 2 on $\left(Q^{*} \mathscr{F}, \widetilde{F}\right)$; that is, the following hold:

(i) $\omega^{i}\left(\xi_{j}\right)=\delta_{j}^{i}, f\left(\xi_{i}\right)=0, \omega^{i} \circ f=0$;

(ii) $f^{2}=-\varepsilon\left(I-\omega^{1} \otimes \xi_{1}-\omega^{2} \otimes \xi_{2}\right)$;

(iii) $f$ is of rank $2 n-2$ and $f^{3}+\varepsilon f=0$.
Theorem 9. The transversal Riemannian metric $G$ on $\left(Q^{*} \mathscr{F}, \widetilde{\mathscr{F}}\right)$ satisfies

$$
\begin{aligned}
G(f(X), f(Y))= & G(X, Y)-\omega^{1}(X) \omega^{1}(Y) \\
& -\omega^{2}(X) \omega^{2}(Y), \quad X, Y \in \Gamma(Q \widetilde{\mathscr{F}}) .
\end{aligned}
$$

For $\varepsilon=1$ we put

$$
\Phi(X, Y)=G(f(X), Y), \quad X, Y \in \Gamma(Q \widetilde{\mathscr{F}}) .
$$

By using Theorems 8 and 9 , we obtain

$$
\Phi(X, Y)=-\Phi(Y, X), \quad X, Y \in \Gamma(Q \widetilde{F}) .
$$

Thus, $\Phi$ is a transverse 2 -form on $\left(Q^{*} \mathscr{F}, \widetilde{\mathscr{F}}\right)$. It is degenerate with null space $\operatorname{span}\left\{\xi_{1}, \xi_{2}\right\}$.

Also, using the calculus in local coordinates, we easily obtain

$$
\begin{gathered}
\Phi\left(\frac{\delta}{\delta x^{a}}, \frac{\delta}{\delta x^{b}}\right)=0, \quad \Phi\left(\frac{\delta}{\delta x^{a}}, \frac{\partial}{\partial p_{b}}\right)=-\delta_{a}^{b}+\frac{p_{a} p^{b}}{K^{2}}, \\
\Phi\left(\frac{\partial}{\partial p_{a}}, \frac{\partial}{\partial p_{b}}\right)=0 .
\end{gathered}
$$

On the other hand, we have

$$
\begin{aligned}
& d \omega^{1}\left(\frac{\delta}{\delta x^{a}}, \frac{\delta}{\delta x^{b}}\right)=\frac{\delta}{\delta x^{a}}\left(\frac{p_{b}}{K}\right)-\frac{\delta}{\delta x^{b}}\left(\frac{p_{a}}{K}\right), \\
& d \omega^{1}\left(\frac{\delta}{\delta x^{a}}, \frac{\partial}{\partial p_{b}}\right)=\frac{1}{K}\left(-\delta_{a}^{b}+\frac{p_{a} p^{b}}{K^{2}}\right), \\
& d \omega^{1}\left(\frac{\partial}{\partial p_{a}}, \frac{\partial}{\partial p_{b}}\right)=0,
\end{aligned}
$$

where in the second relation we have used $p^{a}=K\left(\partial K / \partial p_{a}\right)$ which follows from the homogeneity conditions (18) of $K$. Comparing now $\Phi$ with $d \omega^{1}$ we obtain

$$
\frac{1}{K} \Phi=d \omega^{1}+\Psi
$$

where $\Psi=\left(\left(\delta / \delta x^{b}\right)\left(p_{a} / K\right)-\left(\delta / \delta x^{a}\right)\left(p_{b} / K\right)\right) d x^{a} \wedge d x^{b}$. Thus, $(1 / K) \Phi$ is transversally closed if and only if $\Psi$ is transversally closed. Concluding, $(1 / K) \Phi$ is in general an almost transversally presymplectic structure on $\left(Q^{*} \mathscr{F}, \widetilde{F}\right)$.

Similarly, for $\varepsilon=-1$, we can put

$$
H(X, Y)=G(f(X), Y), \quad X, Y \in \Gamma(Q \widetilde{\mathscr{F}}) .
$$

We have the following.

Theorem 10. The mapping $H$ is a symmetric bilinear form on $\left(Q^{*} \mathscr{F}, \widetilde{\mathscr{F}}\right)$ and the annihilator of $H$ is $\operatorname{ker} f$. 
Proof. The symmetry and bilinearity are obvious. Also, the null space of $H$ is

$$
\begin{aligned}
\{X & \in \Gamma(Q \widetilde{\mathscr{F}}) \mid H(X, Y)=0, \forall Y \in \Gamma(Q \widetilde{\mathscr{F}})\} \\
& =\{X \in \Gamma(Q \widetilde{\mathscr{F}}) \mid G(f(X), Y)=0\}=\operatorname{ker} f
\end{aligned}
$$

which end the proof.

Locally, we obtain

$$
H=\left(g_{a b}-\frac{p_{a} p_{b}}{K^{2}}\right) d x^{a} \otimes d x^{b}-\left(g^{a b}-\frac{p^{a} p^{b}}{K^{2}}\right) \delta p_{a} \otimes \delta p_{b}
$$

with $\operatorname{det}\left(g_{a b}-\left(p_{a} p_{b} / K^{2}\right)\right)=0$, since $\left(g_{a b}-\left(p_{a} p_{b} / K^{2}\right)\right) p^{b}=$ $p_{a}-p_{a}=0$, and similarly $\operatorname{det}\left(g^{a b}-\left(p^{a} p^{b} / K^{2}\right)\right)=0$, since $\left(g^{a b}-\left(p^{a} p^{b} / K^{2}\right)\right) p_{b}=p^{a}-p^{a}=0$.

Remark 11. The map $H$ is a transversally singular pseudoRiemannian metric on $\left(Q^{*} \mathscr{F}, \widetilde{F}\right)$.

\section{An Almost (Para)Contact Structure on Transverse Liouville Distribution of $\left(Q^{*} \mathscr{F}, \widetilde{F}\right)$}

Denote by $\left\{\xi_{2}\right\}$ the line vector bundle over $Q^{*} \mathscr{F}$ spanned by $\xi_{2}$ and we define the transverse vertical Liouville distribution as the complementary orthogonal distribution $S\left(Q^{*} \mathscr{F}\right)$ to $\left\{\xi_{2}\right\}$ in $V\left(Q^{*} \mathscr{F}\right)$ with respect to $G^{v}$; namely, $V\left(Q^{*} \mathscr{F}\right)=S\left(Q^{*} \mathscr{F}\right) \oplus$ $\left\{\xi_{2}\right\}$. Hence, $S\left(Q^{*} \mathscr{F}\right)$ is defined by $\alpha:=\left.\omega^{2}\right|_{V\left(Q^{*} \mathscr{F}\right)}$; that is,

$$
\Gamma\left(S\left(Q^{*} \mathscr{F}\right)\right)=\left\{X \in \Gamma\left(V\left(Q^{*} \mathscr{F}\right)\right) ; \alpha(X)=0\right\} .
$$

Thus, any transverse vertical vector field $X \in \Gamma\left(V\left(Q^{*} \mathscr{F}\right)\right)$ can be expressed as

$$
X=P X+\alpha(X) \xi_{2},
$$

where $P$ is the projection morphism of $V\left(Q^{*} \mathscr{F}\right)$ on $S\left(Q^{*} \mathscr{F}\right)$. By direct calculations, one gets the following.

Proposition 12. For any transverse vertical vector fields $X, Y \in$ $\Gamma\left(V\left(Q^{*} \mathscr{F}\right)\right)$, one has

$$
G^{v}(X, P Y)=G^{v}(P X, P Y)=G^{v}(X, Y)-\alpha(X) \alpha(Y) .
$$

Theorem 13. The transverse vertical Liouville distribution $S\left(Q^{*} \mathscr{F}\right)$ is integrable.

Proof. The proof follows using an argument similar to Theorem 3.1 [14] (see also Theorem 2.1 [6] or Theorem 4 [15]).

In the following, we will consider the transverse Liouville distribution of $\left(Q^{*} \mathscr{F}, \widetilde{F}\right)$ as the complementary orthogonal distribution $\bar{S}\left(Q^{*} \mathscr{F}\right)$ to $\left\{\xi_{2}\right\}$ in $Q \widetilde{\mathscr{F}}$ with respect to $G$; that is, $\bar{S}\left(Q^{*} \mathscr{F}\right)=H\left(Q^{*} \mathscr{F}\right) \oplus S\left(Q^{*} \mathscr{F}\right)$.

Let us restrict to $\bar{S}\left(Q^{*} \mathscr{F}\right)$ all the geometrical structures introduced in Section 4 for all $Q \widetilde{\mathscr{F}}$. We indicate this by overlines. Hence, we have (i) $\overline{\xi_{1}}=\xi_{1}$ since $\xi_{1}$ lies in $\bar{S}\left(Q^{*} \mathscr{F}\right)$;

(ii) $\overline{\omega^{2}}=0$ since $\omega^{2}(X)=G\left(X, \xi_{2}\right)=0$ for every transverse vector field $X \in \bar{S}\left(Q^{*} \mathscr{F}\right)$;

(iii) $\bar{G}=\left.G\right|_{\bar{S}\left(Q^{*} \mathscr{F}\right)}$;

(iv) $\bar{f}(X)=\bar{\phi}(X)+\varepsilon \overline{\omega^{1}}(X) \otimes \xi_{2}$ is an endomorphism of $\bar{S}\left(Q^{*} \mathscr{F}\right)$ since

$$
\begin{aligned}
G\left(\bar{f}(X), \xi_{2}\right) & =G\left(\bar{\phi}(X), \xi_{2}\right)+\varepsilon \overline{\omega^{1}}(X) G\left(\xi_{2}, \xi_{2}\right) \\
& =\omega^{2}(\bar{\phi}(X))+\varepsilon \overline{\omega^{1}}(X)=0 .
\end{aligned}
$$

We denote now $\bar{\xi}=\overline{\xi_{1}}$ and $\bar{\eta}=\overline{\omega^{1}}$.

By Theorem 8, we obtain the following.

Theorem 14. The triple $(\bar{f}, \bar{\xi}, \bar{\eta})$ provides an almost (para)contact structure on $\bar{S}\left(Q^{*} \mathscr{F}\right)$; that is,

(i) $\bar{f}^{3}+\varepsilon \bar{f}=0, \operatorname{rank} \bar{f}=2 n-2=(2 n-1)-1$;

(ii) $\bar{\eta}(\bar{\xi})=1, \bar{f}(\bar{\xi})=0, \bar{\eta} \circ \bar{f}=0$;

(iii) $\bar{f}^{2}(X)=-\varepsilon(X-\bar{\eta}(X) \bar{\xi})$, for $X \in \bar{S}\left(Q^{*} \mathscr{F}\right)$.

Also, by Theorem 9 we obtain the following.

Theorem 15. The transversal Riemannian metric $\bar{G}$ verifies

$$
\bar{G}(\bar{f}(X), \bar{f}(Y))=\bar{G}(X, Y)-\bar{\eta}(X) \bar{\eta}(Y),
$$

for every transverse vector fields $X, Y \in \bar{S}\left(Q^{*} \mathscr{F}\right)$.

Concluding, the ensemble $(\bar{f}, \bar{\xi}, \bar{\eta}, \bar{G})$ is an almost (para)contact Riemannian structure on $\bar{S}\left(Q^{*} \mathscr{F}\right)$.

\section{Conflict of Interests}

The authors declare that they have no conflict of interests.

\section{Authors' Contribution}

Both authors contributed equally to the paper. All the authors read and approved the final paper.

\section{Acknowledgment}

The first author is supported by the Grant "Fellowship of Transilvania University of Braşov 2014."

\section{References}

[1] A. Miernowski and W. Mozgawa, "Lift of the Finsler foliation to its normal bundle," Differential Geometry and Its Applications, vol. 24, no. 2, pp. 209-214, 2006.

[2] P. Popescu and C. Ida, "Foliations compatible with hamiltonians," in Proceedings of the BSG International Conference on Differential Geometry and Dynamical Systems (DGDS '13), pp. 147-155, Bucharest, Romania, October 2013. 
[3] P. Popescu and M. Popescu, "Lagrangians adapted to submersions and foliations," Differential Geometry and Its Applications, vol. 27, no. 2, pp. 171-178, 2009.

[4] P. Popescu and M. Popescu, "Foliated vector bundles and Riemannian foliations," Comptes Rendus Mathematique, vol. 349, no. 7-8, pp. 445-449, 2011.

[5] P. Popescu and M. Popescu, "Lagrangians and Hamiltonian structures and foliations," Physics AUC, vol. 21, no. 1, pp. 203206, 2011.

[6] C. Ida, "Some framed $f$-structures on transversally Finsler foliations," Annales Universitatis Mariae Curie-Sklodowska, Sectio A: Mathematica, vol. 65, no. 1, pp. 87-96, 2011.

[7] M. Abate and G. Patrizio, Finsler Metrics-A Global Approach, vol. 1591 of Lecture Notes in Mathematics, Springer, Berlin, Germany, 1994.

[8] R. Miron, D. Hrimiuc, H. Shimada, and S. V. Sabău, The Geometry of Hamilton and Lagrange Spaces, vol. 118 of Fundamental Theories of Physics, Kluwer Academic, 2001.

[9] K. Yano, "On a structure defined by a tensor field of type $(1,1)$ satisfying $f^{3}+f=0$," Tensor, vol. 14, pp. 99-109, 1963.

[10] M. Anastasiei, "A framed f-structure on tangent bundle of a Finsler space," Analele Universitatii Bucuresti, Seria Matematica, vol. 49, pp. 3-9, 2000.

[11] M. Gîrtu, "An almost 2-paracontact structure on the cotangent bundle of a Cartan space," Hacettepe Journal of Mathematics and Statistics, vol. 33, pp. 15-22, 2004.

[12] M. Gîrtu, "A Sasakian structure on the indicatrix bundle of a Cartan space," Analele Ştiinţifice ale Universitatii "Al.I.Cuza” din Iasi, Tomul LIII, vol. 1, pp. 177-186, 2007.

[13] I. Mihai, R. Rosca, and L. Verstraelen, Some Aspects of the Differential Geometry of Vector Fields, vol. 2 of Centre for Pure and Applied Differential Geometry (PADGE), Katholieke Universiteit Leuven, Leuven, Belgium, 1996.

[14] A. Bejancu and H. R. Farran, "On the vertical bundle of a pseudo-Finsler manifold," International Journal of Mathematics and Mathematical Sciences, vol. 22, no. 3, pp. 637-642, 1999.

[15] C. Ida and A. Manea, "A vertical Liouville subfoliation on the cotangent bundle of a Cartan space and some related structures," International Journal of Geometric Methods in Modern Physics, vol. 11, no. 6, 21 pages, 2014. 


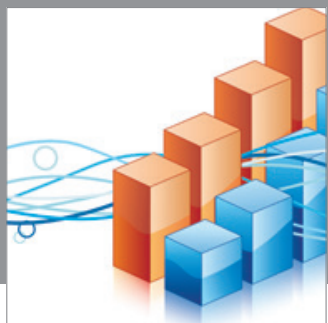

Advances in

Operations Research

mansans

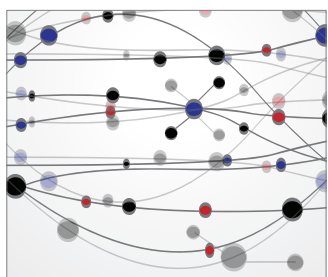

The Scientific World Journal
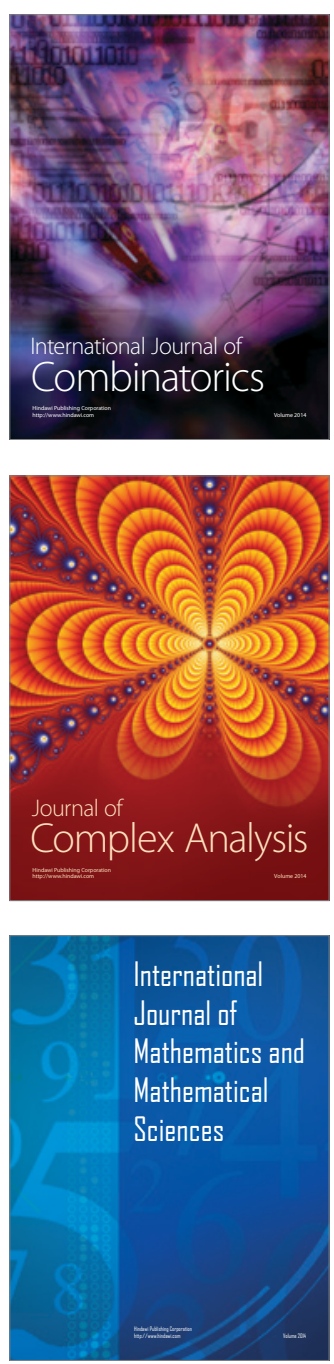
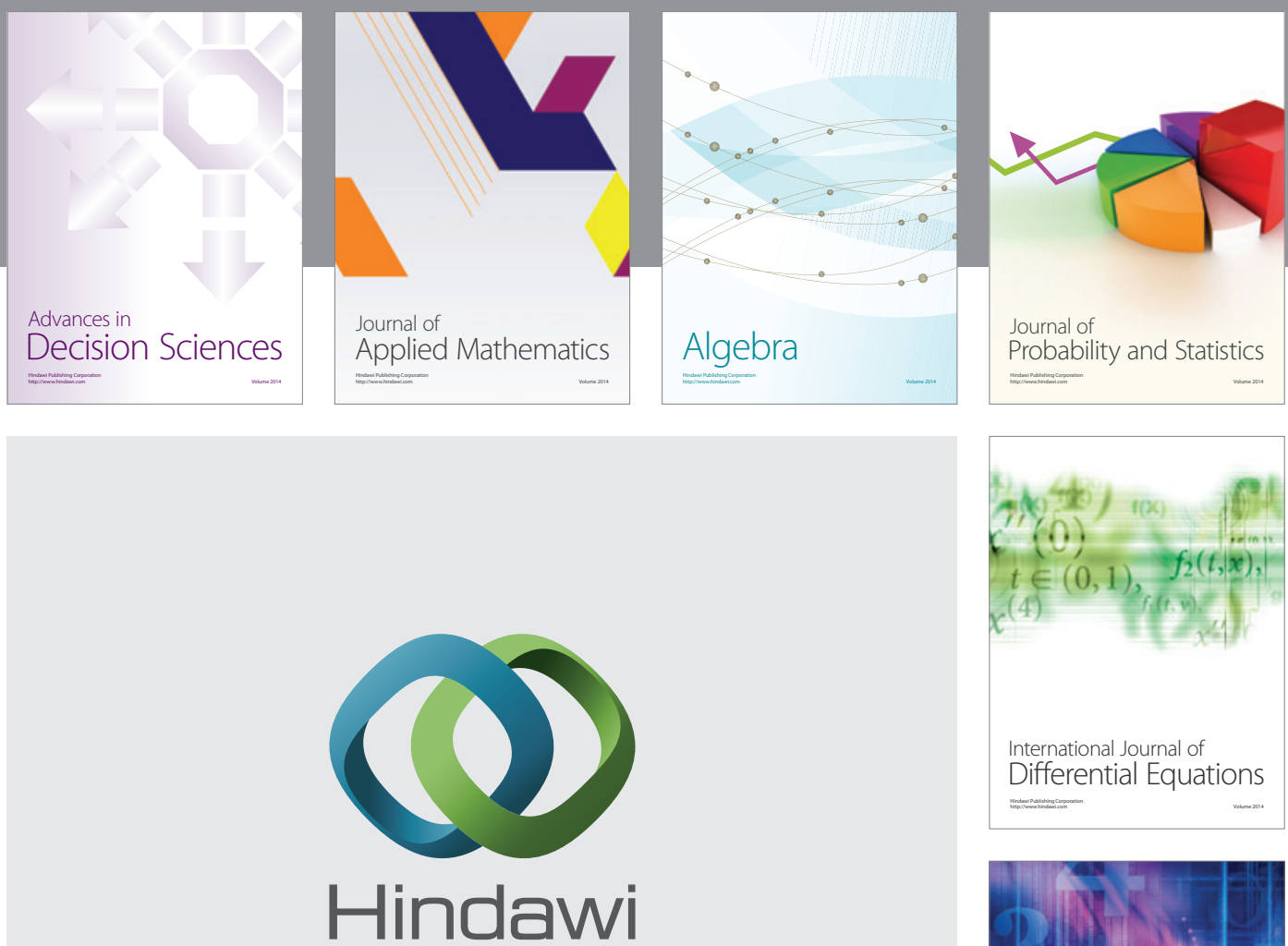

Submit your manuscripts at http://www.hindawi.com
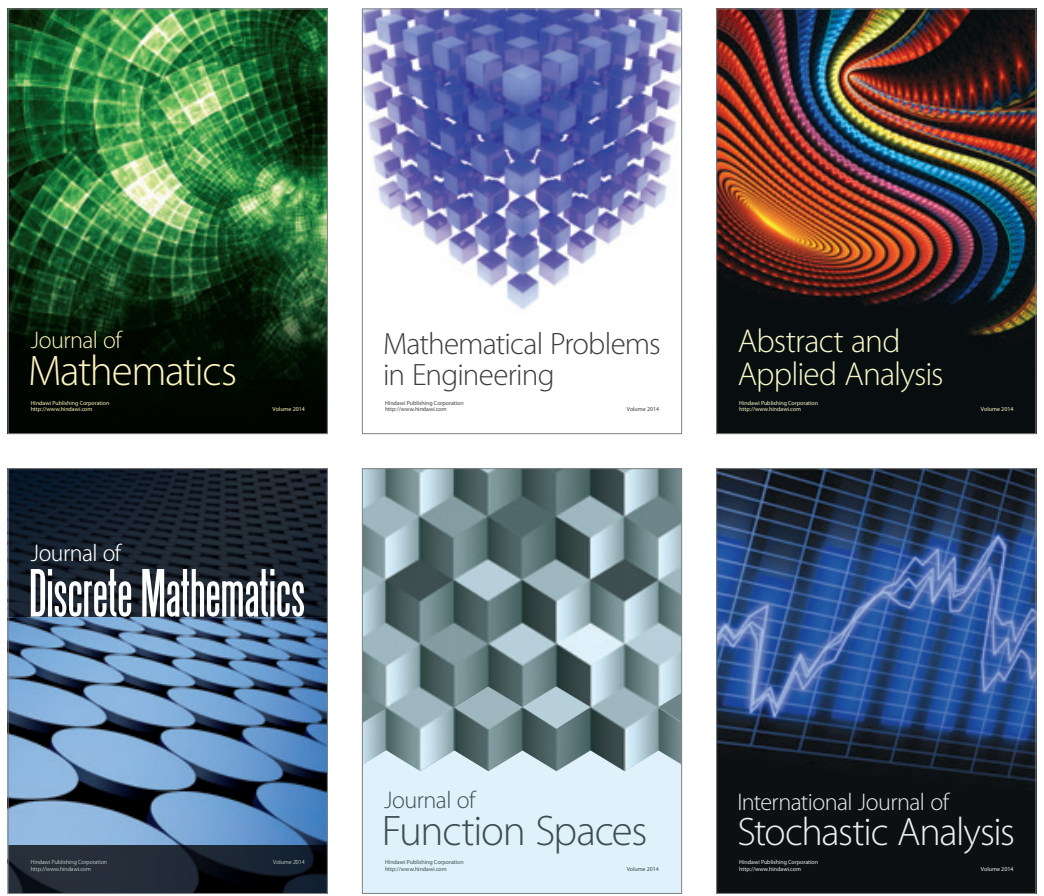

Journal of

Function Spaces

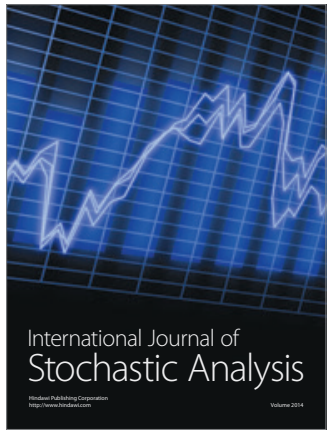

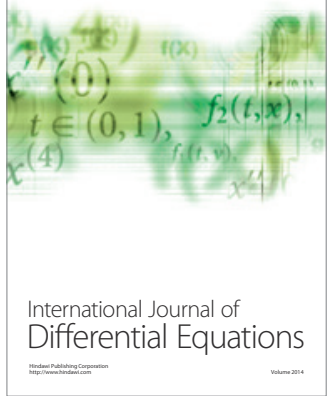
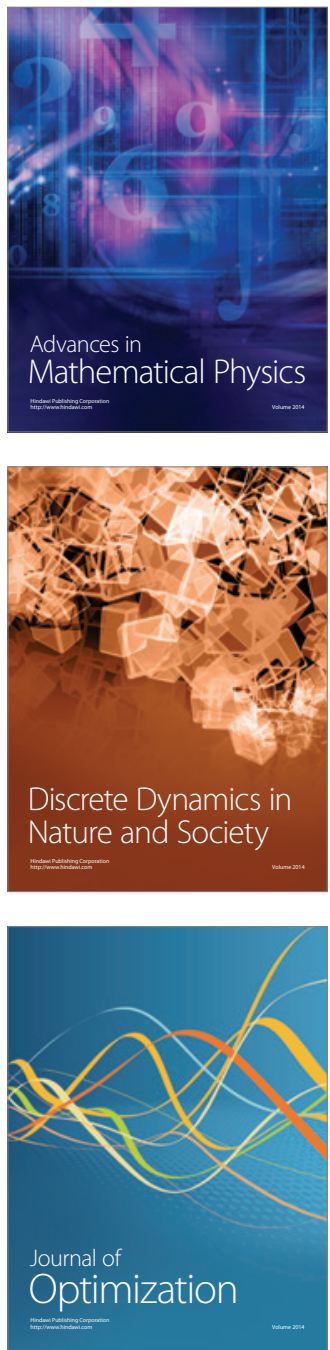